\title{
Going Rogue: What scientists can learn about Twitter communication from "alt" government accounts
}

\author{
Matthew J. Wilson ${ }^{\text {Corresp., } 1}$, Elizabeth K. Perkin ${ }^{2}$ \\ ${ }^{1}$ Freshwater Research Institute, Susquehanna University, Selinsgrove, Pennsylvania, United States \\ 2 Native Fish Society, Oregon City, Oregon, United States \\ Corresponding Author: Matthew J. Wilson \\ Email address: wilsonmatt@susqu.edu
}

The inauguration of President Trump in the United States led to the active restriction of science communication from federal agencies, resulting in the creation of many unofficial "alt" Twitter accounts to maintain communication. Alt accounts had many followers (e.g., 15 accounts had $>100,000$ ) and received a large amount of media attention, making them ideal for better understanding how differences in messaging can affect public engagement with science on microblogging platforms. We analyzed tweets produced by alt and corresponding official agency accounts to compare the two groups and determine if specific features of a tweet made them more likely to be retweeted or liked to help the average scientist potentially reach a broader audience on Twitter. We found adding links, images, hashtags, and mentions, as well as expressing angry and annoying sentiments all increased retweets and likes. Evidence-based terms such as "peer-review" had high retweet rates but linking directly to peer-reviewed publications decreased attention compared to popular science websites. Word choice and attention did not reflect official or alt account types, indicating topic is more important than source. The number of tweets generated and attention received by alt accounts has decreased since their creation, demonstrating the importance of timeliness in science communication on social media. Together our results show potential pathways for scientists to increase efficacy in Twitter communications. 


\section{Going Rogue: What scientists can learn about Twitter} 2 communication from "alt" government accounts

3

4 Matthew J. Wilson ${ }^{1}$ and Elizabeth K. Perkin ${ }^{2}$

5

$6 \quad{ }^{1}$ Freshwater Research Institute, Susquehanna University, Selinsgrove, Pennsylvania, USA

$7 \quad{ }^{2}$ Native Fish Society, Oregon City, Oregon, USA

8

9 Corresponding Author:

10 Matthew Wilson ${ }^{1}$

11514 University Avenue, Selinsgrove, Pennsylvania, 17870, USA

12 Email address: wilsonmatt@susqu.edu 


\section{Abstract}

15 The inauguration of President Trump in the United States led to the active restriction of science

16 communication from federal agencies, resulting in the creation of many unofficial "alt" Twitter

17 accounts to maintain communication. Alt accounts had many followers (e.g., 15 accounts had

$18>100,000)$ and received a large amount of media attention, making them ideal for better

19 understanding how differences in messaging can affect public engagement with science on

20 microblogging platforms. We analyzed tweets produced by alt and corresponding official agency

21 accounts to compare the two groups and determine if specific features of a tweet made them

22 more likely to be retweeted or liked to help the average scientist potentially reach a broader

23 audience on Twitter. We found adding links, images, hashtags, and mentions, as well as

24 expressing angry and annoying sentiments all increased retweets and likes. Evidence-based terms

25 such as "peer-review" had high retweet rates but linking directly to peer-reviewed publications

26 decreased attention compared to popular science websites. Word choice and attention did not

27 reflect official or alt account types, indicating topic is more important than source. The number

28 of tweets generated and attention received by alt accounts has decreased since their creation,

29 demonstrating the importance of timeliness in science communication on social media. Together

30 our results show potential pathways for scientists to increase efficacy in Twitter

31 communications.

32

33 Key Words

34 Social media, text sentiment, word choice, mixed models, targeted outreach, science

35 communication, Web 2.0 


\section{INTRODUCTION}

38

39

40

41

42

43

44

45

46

47

48

49

50

51

52

53

54

55

56

57

58

59

60

61

62

63

64

65

66

67

68

69

70

71

72

73

74

75

76

Since its inception in 2006, the microblogging social media platform Twitter has dramatically changed the science communication landscape, allowing scientists to communicate directly with a broad audience as well as one another. The abilities to directly comment to politicians on science policy issues and potentially influence media coverage are particular perks of this method of communication (Newman 2016). However, many scientists create tight networks across media platforms where they primarily communicate with other scientists (Wilson et al. 2016) which can particularly undermine the potential of social media platforms like Twitter to reach large audiences and implement real-world change (Letierce et al. 2010). For instance, in one survey of scientists, $47 \%$ of researchers who used social media to share their findings had been contacted by other researchers, while only $28 \%$ had been contacted by members of the public as a result of this outreach (Wilkinson and Weitkamp 2013). Conversely, when scientists are able to expand their network of followers it has the potential to not only increase their citation rate (Peoples et al., 2016; Lamb C, Gilbert SL, \& Ford, 2018), but also readership among the media and general public (Côté \& Darling, 2018), demonstrating the value of social media platforms when used effectively. Further, analyses of microblogging data have improved our understanding of how topics such as conservation awareness and media coverage (Acerbi et al., 2020), common vs Latin species name use (Jarić et al., 2016), and informal citizen science (Daume \& Galaz, 2016) reach lay audiences.

Government agencies involved with science and policy also seek to communicate scientific information to a broad audience. However, following the 2017 inauguration of President Trump, the mandate of government agencies within the United States to communicate scientific findings was questioned by the executive branch of the government, including the prohibition of official accounts from tweeting climate-related information (Volcovici and Huffstutter 2017). Employees within many of these U.S. agencies have created "alt" or "rogue" (henceforth referred to as "alt") Twitter accounts as a way to continue to share information as well as criticize the current presidential administration within the United States (Davis 2017).

These alt Twitter accounts gained coverage across a wide variety of journalistic platforms, from blogs (e.g., LiveScience: Weisberger 2017) to cable news (e.g., CNN: Walker 2017) and developed large numbers of followers. Because these alt accounts had more followers than most individual scientists, we were interested in determining if the scientific community might gain valuable insight for better reaching and broadening their Twitter audience by studying the habits of alt and corresponding official Twitter accounts of U.S. agencies. Specifically, we were interested in what topics receive the most attention in alt versus official accounts, if there is a large discrepancy between the hashtags and mentions between alt and official accounts, what types of keywords and links (e.g. peer-reviewed papers or popular science websites) garner the most attention, and how attention to alt accounts changed over time. We limited our study to focus on agencies involved in conservation biology, as we anticipated that

Peer) reviewing PDF | (2021:05:61059:1:1:NEW 6 Sep 2021) 
77 patterns in hashtags, links and keywords would be more readily apparent by focusing on one area

78 of science.

\section{MATERIALS AND METHODS}

80 Data Collection

81 We identified all official U.S. federal Twitter accounts with an "alt" or "rogue" corollary

82 handle that could be linked with a single federal agency and collected total followers, tweets,

83 likes, and accounts followed for each account on 1 April 2017 by searching Twitter with the

84 terms "rogue" and "alt" then identifying the official account that was the intended target of each

85 alt account (Table S1). To identify additional unofficial accounts under other titles (e.g.

$86 @$ @BadHombreNPS) we scanned followers of the unofficial accounts previously identified with

87 the assumption that alt accounts would form networks with each other. We collected original

88 tweets for alt accounts since their creation (generally 24 Jan 2017), and tweets between 1 Jan

892016 and1 April 2017 for official accounts, using the Java project GetOldTweets to avoid date

90 constraints of the Twitter Application Programming Interface (Henrique 2016). These tweets

91 were collected two weeks after the final date posted (14 April 2017) to decrease the likelihood

92 total retweets and likes of the most recent tweets were underestimated (popular tweets might be

93 liked and retweeted for several days after posting; Lou et al. 2015). All tweets were also

94 collected within a three hour period to limit differences in retweet and like numbers further, as

95 new retweets or likes could be registered during collection. On 1 April 2017, there were 120

96 active alt accounts that could be linked directly to 71 official Twitter accounts of the U.S.

97 government (Table S1).

98

99

100

101

102

103

104

105

106

107

108

109

110

111

112

113

114

115

116

We selected only those official-alt account pairs in which the most prominent alt account had at least 50,000 followers on 1 April 2017. From these criteria we selected the official and top alt account pairs for National Parks Service (NPS), National Oceanic and Atmospheric Administration (NOAA), U.S. Environmental Protection Agency (USEPA), U.S. Department of Agriculture (USDA), U.S. Forest Service (USFS), and U.S. Fish and Wildlife Service (USFWS). We also included the official and alt accounts for Badlands National Park, as the alt account for this park had more followers $(209,000)$ than the alt account for NPS $(89,000)$. The primary alt account for NPS changed names on 29 Jan 2017. We included both handles (@NotAltWorld and @AltNatParkSer) and their corresponding tweets separately in analyses by account to avoid confounding results by the change in followers corresponding to this name change. With the inclusion of both NPS alt handles, the dataset included 15 handles and 9,688 tweets.

\section{Tweet Emotion and Features}

To examine the potential effects of tweet emotion on attention (measured as retweets and likes) we assigned emotions to each tweet with the DepecheMood lexicon database, based on social news media with high lexical precision (Staiano and Guerini 2014). This high-coverage database characterizes 37,000 English terms by seven possible emotions: afraid, amused, angry, annoyed, happy, inspired, and sad. Each term in the database is associated with all emotions on a 
$1170-1$ scale so that the combined value for all emotions conveyed by a single term is 1 . To give 118 overall emotions to tweets, we applied the normalized DepecheMood database to each tweet and 119 used the mean value for all emotions across all terms in the tweet to represent overall emotion.

120 We selected DepecheMood for the high coverage of terms and large corpus, as well as the ease 121 of access and implementation for broad applicability. See Giachanou and Crestani (2016) for a 122 detailed review of Twitter sentiment methods.

123

124

125

126

127

128

129

130

131

132

133

134

135

136

137

138

139

140

141

142

143

144

145

146

147

148

149

150

151

152

153

154

155

156

\section{Science Terms and Retweets}

To determine if specific terms increased the likelihood of attention, we created a list of science- and evidence-based terms used in tweets, by splitting all tweets into individual terms (words within tweet text and hashtags) to compare with the associated number of retweets and averaged retweets by the associated term frequency. We manually identified all unambiguous science and evidence related terms used in tweets (e.g., "vulture" and "rat", which were often used to vilify people and/or other agencies, were excluded) and merged singular and plural versions of terms into a single value. We excluded agency related terms (e.g., \#usda or \#epa) from the final matrix. This left a term by count and average retweet matrix of 496 terms (Table S2). For comparisons of this term matrix by account type (pre, post, alt) and agency we used Canonical Correspondence Analysis (CCA) to deal with the unbalanced sample size across groups (e.g. high numbers of tweets from alt compared to official accounts). Agency and account type were tested for significant effects on overall science term use and term retweets via ANOVA-like permutations (ter Braak and Verdonschot 1995, Legendre et al. 2011) with the vegan package in $\mathrm{R}$ (Oksanen et al. 2017; R Core Team 2016). While not used for analysis, we 
157 categorized the science term matrix further (e.g., by "ecosystem", "evidence", "organism") to

158 identify which terms within categories received the most attention and how categories were

159 associated with CCA results. To be conservative, terms ambiguously within multiple categories

160 were left uncategorized (Table S2).

161

162

163

164

165

166

167

168

169

170

171

172

\section{Tweet Links}

We investigated the effect of linking to science-specific websites in tweets compared to other link types and divided these into direct peer-review links to publications and links to popular science websites. Tiny URLs attached to tweets were decoded by the stand-alone "decode_short_url" R function (Breyal 2012) and manually verified. The majority of peer-review links were tweeted by @AltUSEPA (15 of 22 tweets), therefore we restricted our analysis of lay and peer-reviewed links to this account. We compared retweets and likes between groups with a Kruskal-Wallis ranked ANOVA for multiple comparisons corrected for ties with the PMCMR package (Pohlert 2014; Canover and Iman 1979).

173

174

\section{Time Series}

To test the significance of timeliness in tweet attention, we performed multiple linear

175 regression of total retweets, retweets per tweet, total likes, and likes per tweet against the number

176

177 of days after creation of the first alt account, grouped by alt and official accounts. We excluded the date the first alt account was created (24 Jan 2017) to remove the skewing effects (i.e., very

178 high attention on Twitter) of tweets made by all accounts on that date. All reported r-squared values are adjusted r-squared.

179

180

181

182

183

184

185

186

187

188

189

190

191

192

193

\section{RESULTS}

\section{Tweet Emotion and Features}

Most emotions did not significantly affect retweets or likes. However, tweets that conveyed anger significantly increased both retweets and likes, annoying language significantly increased likes, and inspired language significantly decreased both retweets and likes. Angry language also had the strongest effect on number of retweets and likes. Higher numbers of hashtags and mentions and the attachment of images or links to tweets all significantly increased both retweets and likes. These effects were smaller than significant emotions and also had smaller confidence intervals (i.e. more consistent effects) than emotions. There were no significant effects of account type (official-pre, official-post, or alt; Table 1).

\section{Science Terms and Retweets}

Canonical Correspondence Analysis results showed a significant effect of U.S. agency on science-related word and hashtag use in tweets (adjusted $\mathrm{R}^{2}=0.14, \mathrm{p}<0.001$ ). While there was

194

195 some visible separation of word choice by account type (pre, post, and alt), this relationship was not significant (Figure 1A). This pattern was primarily the result of higher use of terms related to

196 chemistry, evidence, fossil fuel, pollution, and climate by alt accounts, as well as NOAA and 
197 USEPA accounts. When looking at the results by agency, differences were most visible along

198

199

200

201

202

203

204

205

206

207

208

209

210

211

212

213

214

215

216

217

218

219

220

221

222

223

224

225

226

227

228

229

230

231

232

233

234

235

236

Axis 2, with tweets from the USFWS accounts containing more organisms (particularly birds, reptiles, and amphibians) while activities (e.g., camping, fishing, hiking) were most closely related to tweets from USFS accounts (Figure 1B). Neither grouping accounts by agency nor by type had a significant effect on retweets of science terms. The effect of agency on retweets by word were negligible $\left(\mathrm{R}^{2}<0.05\right)$. Words and hashtags that received the most attention for climate change-related terms were related to current fossil fuel extraction (e.g., "keystone" and "spill"), "peer-review" and "conferences" were the most retweeted evidence terms, flowers and large mammals were the most retweeted organism groups, and prairies and forests were the top retweeted ecosystems (Table 2).

\section{Tweet Links}

For tweets by @altUSEPA, the addition of website links significantly increased both retweets $(99.6 \pm 7.1, \mathrm{p}<0.0001, \mathrm{n}=522)$ and likes $(173.4 \pm 12.9, \mathrm{p}<0.0001, \mathrm{n}=522)$ over tweets without links $(19.9 \pm 2.4$ retweets/tweet and $73.0 \pm 8.6$ likes/tweet, $n=1374)$. This increase in attention was further amplified by the use of links to popular science websites (127.4 \pm 32.0 retweets/tweet and $232.0 \pm 59.3$ likes/tweet; $\mathrm{n}=52$ and $\mathrm{p}<0.0001$ for both retweets and likes) compared to tweets without links. However, linking to peer-reviewed publications rather than popular science significantly decreased the number of retweets $(44.2 \pm 18.4, p=0.02, n=$ $15)$ and likes $(78.1 \pm 35.7, \mathrm{p}=0.032, \mathrm{n}=15)$ compared to other websites and was not significantly different from tweets without links.

\section{Time Series}

Tweets from alt accounts showed a significant decrease in total retweets $\left(R^{2}=0.13, p=\right.$ $0.002)$, retweets per tweet $\left(R^{2}=0.16, p=0.0005\right)$, total likes $\left(R^{2}=0.12, p=0.002\right)$, and likes per tweet $\left(R^{2}=0.15, p=0.0008\right)$ between 25 Jan 2017 and 1 April 2017 (Figure 2). None of the attention metrics tested for official accounts had significant temporal trends (all $\mathrm{R}^{2}<0.03$ ).

\section{DISCUSSION}

By closely examining tweets from both alt and official government accounts, we were able to discover underlying variables within the tweets that could help predict if a tweet would be liked or retweeted. We were surprised to see tweets categorized as "angry" were both significantly more likely to be retweeted and to be liked, as Hutto, Yardi, and Gilbert (2013) found negative sentiment in tweets to have a negative effect on the number of followers accrued over time. One possible explanation for these opposing findings might be a general sentiment of anger felt by people who support these government accounts at this point in time and may not represent the best tactic for most scientists to increase outreach. Exceptions might be when one wants to take advantage of a particularly emotionally charged event. However, leveraging emotion, especially politically-motivated anger, could actually result in these alt accounts reinforcing ideas that scientists are elitists and work only for Democrats, while further alienating 
237 Republicans (Nisbet and Scheufele 2009). On the other hand, the finding that tweets containing

238 hashtags, mentions, images, and links (images and links in particular) accumulate more retweets

239 and likes, is a strategy that all scientists can easily incorporate into their tweeting practices in

240 order to increase their reach and mirrors results from U.S. health agencies (Bhattacharya,

241 Srinivasan, \& Polgreen, 2014). This finding could also be valuable for scientists in gaining trust

242 via Twitter as tweets containing links have been viewed as more credible, regardless of the

243 linked website (Aigner et al. 2017).

244

245

246

247

248

249

250

251

252

253

254

255

256

257

258

259

260

261

262

263

264

265

266

267

268

269

270

271

272

273

274

275

276

The terms used in tweets that received the most retweets were generally evidence- or climate-based. Words including "peer reviewed," "knowledge," and "verify" were associated with extremely high levels of retweets per tweet. This suggests researchers should feel comfortable using common science-based terms and that a large audience supports these terms. While ecosystem- and organism-specific terms did not receive as many retweets as those tweets containing evidence-based or climate-specific terms, there were still some important trends to emerge out of those categories, with ecosystems that people frequently come into contact with (e.g., prairie, forest, streams), and flowers and large mammals (e.g., violets, bison, wolf) leading to higher numbers of retweets. Similar patterns emerged in previous research examining which species listed under the Endangered Species Act were most tweeted (Roberge 2014). While there were themes in the most common categories tweeted by alt accounts that might be expected (e.g., fossil fuel, evidence, and climate), our CCA analysis revealed that word choice in tweets was strongly determined by agency (e.g., NOAA, USFS), but not by type (pre, post, alt), suggesting alt accounts might really be run by agency personnel, as claimed.

Given the number of retweets of tweets containing evidence-based terms, particularly those tweets containing the phrase "peer reviewed," it surprised us that tweets containing links to actual peer reviewed papers were less likely to be retweeted or liked than those with other links. This suggests that while it can be tempting to share and promote peer reviewed literature on Twitter, this is not the best way to reach a broader audience. Rather, scientists interested in reaching a broader audience would be well advised to reach out to traditional media (Nisbet and Scheufele 2009) and then share links to those "interpreted" stories. Indeed, we found tweets containing links to popular science articles significantly increased the likelihood that a tweet would be liked or retweeted. Interestingly, Holmberg and Thelwall (2014) found that scientists rarely use links when discussing a topic with one another on Twitter, but based on our findings, researchers may want to change this habit if they would like to expand their audience.

Perhaps one of our most important results was finding how timeliness plays a role in public interest in a topic. The number of retweets per tweet for both official and alt government accounts peaked soon after the inauguration of President Trump, when public interest in these government agencies was extremely high. However, very soon after, interest in all accounts returned to background levels. As a result, it might be important for scientists to take advantage 
277 of large, well-publicized events to tweet important information in order to reach a broad,

278 interested, and engaged audience. When analyzing the popularity of several scientific terms on

279 Twitter, Uren and Dadzie (2015) found similar results, with very high levels of interest in

280 "curiosity" in the few days surrounding the successful landing of the Mars Curiosity rover and

281 on the anniversary of the landing a year later, but with very low levels of interest beyond that

282 time-frame.

283

284

285

286

287

288

289

290

291

292

293

294

295

296

297

298

299

300

301

302

303

304

305

306

307

308

309

310

\section{Acknowledgements}

312 We thank federal U.S. employees for maintaining scientific integrity, perseverance, defiance, and 313 creativity in continued science communication with the public. We particularly thank those

314 anonymous individuals who began the alt movement on social media. This manuscript was

315 improved by comments from A. M. Chará-Serna, B. W. Kielstra, and T. K. Muenz, M.

316 Takahashi, and three anonymous reviewers. 


\section{References}

319

320

321

322

323

324

325

326

327

328

329

330

331

332

333

334

335

336

337

338

339

340

341

342

343

344

345

346

347

348

349

350

351

352

353

354

355

356

357

Acerbi A, Kerhoas D, Webber AD, McCabe G, Mittermeier RA, Schwitzer C. 2020. The impact of the "World's 25 Most Endangered Primates" list on scientific publications and media. Journal for Nature Conservation, 54:125794

Aigner J., A. Durchardt, T. Kersting, M. Kattenbeck, and D. Elsweiler. 2017. Manipulating the perception of credibility in refugee related social media posts. In: Proceedings of the conference human information interaction and retrieval. Organised by Nordlie R., and N. Pharo. Oslo, Norway: ACM. March 7-11, 2017. Pp. 297-300.

Bhattacharya S., P. Srinivasan, and P. Polgreen. 2014. Engagement with health agencies on twitter. PLoS One 9(11): e112235.

Breyal T. 2012. R Function: decode_shortened_url [online]: Available from https://github.com/tonybreyal/Blog-ReferenceFunctions/tree/master/R/decode shortened url.

Burnham K, and D. Anderson. 2002. Model selection and multi-model inference: a practical information theoretic approach. New York, NY: Springer

Conover W.J., and R.L. Iman. 1979. On multiple-comparisons procedures. Los Alamos Scientific Laboratory, Tech. Rep. LA-7677-MS. 17 p.

Cooke S.J., A.J. Gallagher, N.M. Sopinka, V.M. Nguyen, R.A. Skubel, N. Hammerschlag, S.

Boon, N. Young, and A.J. Danylchuk. 2017. Considerations for effective science communication. FACETS 2: 233-248.

Côté IM, Darling ES. 2018. Scientists on Twitter: Preaching to the choir or singing from the rooftops? FACETS 3:682-694.

Daume S, Galaz V. 2016. "Anyone know what species this is?"- Twitter conversations as embryonic citizen science communities. PloS One 11 DOI: 10.1371/journal.pone. 0151387

Davis W. 2017. It's not just the Park Service: 'Rogue' federal Twitter accounts multiply [online]. National Public Radio. Available from http://www.npr.org/sections/alltechconsidered/2017/01/27/512007632/its-not-just-thepark-service-rogue-federal-twitter-accounts-multiply.

Giachanou A, Crestani F. 2016. Like it or not: A survey of twitter sentiment analysis methods. ACM Computing Surveys (CSUR) 49:1-41.

Henrique J. 2016. Get Old Tweets Programatically - Java Project [online]: Available from https://github.com/Jefferson-Henrique/GetOldTweets-java.

Holmberg K., and M. Thelwall. 2014. Disciplinary differences in Twitter scholarly communication. Scientometrics 101(2): 1027-1042.

Hutto C.J., S. Yardi, and E. Gilbert. 2013. A longitudinal study of follow predictors on twitter. In: Proceedings of the SIGCHI conference on human factors in computing systems. Organised by Mackay W. Paris: ACM April 27-May 2, 2013. Pp. 821-830.

Jarić I, Courchamp F, Gessner J, Roberts DL. 2016. Data mining in conservation research using Latin and vernacular species names. PeerJ 4 DOI: 10.7717/peerj.2202.

Peer) reviewing PDF | (2021:05:61059:1:1:NEW 6 Sep 2021) 
358

359

360

361

362

363

364

365

366

367

368

369

370

371

372

373

374

375

376

377

378

379

380

381

382

383

384

385

386

387

388

389

390

391

392

393

394

395

396

397

398

Lamb C, Gilbert SL, Ford AT. 2018. Tweet success? Scientific communication correlates with increased citations in Ecology and Conservation. PeerJ 6 DOI: 10.7717/peerj.4564.

Legendre P, J, Oksanen, and C.J.F. ter Braak. 2011. Testing the significance of canonical axes in redundancy analysis. Methods in Ecology and Evolution 2(3):269-277.

Letierce J, A. Passant, S. Decker, and J.G. Breslin. 2010. Understanding how Twitter is used to spread scientific messages. In: Proceedings of the WebSci10: Extending the frontiers of society on-line. Organised by Hall W., J. Hendler, C. Lagoze, C. Pope, A. T. Schreiber. Raleigh, North Carolina. April 26-27, 2010. Pp. 1-8.

Luo Z, Y. Wang, X. Wu, W. Cai, and T. Chen. 2015. On burst detection and prediction in retweeting sequence. In: Advances in Knowledge Discovery and Data Mining: PAKDD 2015. Lecture Notes in Computer Science. (eds. Cao, T., E.P. Lim, Z.H. Zhou, T.B. Ho, D. Cheung, and H. Motoda. Volume 9077. Pp $1-12$.

Newman T.P. 2016. Tracking the release of IPCC AR5 on Twitter: Users, comments, and sources following the release of the Working Group I Summary for Policymakers. Public Understanding of Science 26(7): 815-825.

Nisbet M.C., and D.A. Scheufele. 2009. What's next for science communication? Promising directions and lingering distractions. American Journal of Botany 96(10): 1767-1778.

Oksanen J, F.G. Blanchet, M. Friendly, R. Kindt, P. Legendre, D. McGlinn, P.R. Minchin, R.B. O'Hara, G.L. Simpson, P. Solymos, M.H.H. Stevens, E. Szoecs, H. Wagner vegan: Community Ecology Package [online]. R package, Version 2.4-3: Available from https://CRAN.R-project.org/package=vegan.

Peoples BK, Midway SR, Sackett D, Lynch A, Cooney PB. 2016. Twitter predicts citation rates of ecological research. PloS One 11 DOI: 10.1371/journal.pone.0166570.

Pohlert, T. 2014. The Pairwise Multiple Comparison of Mean Ranks Package (PMCMR) [online]. R package: Available from http://CRAN.R-project.org/package=PMCMR.

R Core Team. 2016. R: A language and environment for statistical computing. R Foundation for Statistical Computing, Vienna, Austria.

Roberge J.M. 2014. Using data from online social networks in conservation science: which species engage people the most on Twitter? Biodiversity and Conservation 23(3): 715 726.

Staiano J., M. and Guerini. 2014. DepecheMood: a lexicon for emotion analysis from crowdannotated news. In: Proceedings of the 52nd annual meeting of the association for computational linguistics. Organised by NAMES. Baltimore, Maryland: PUBLISHER June DATE 2014. Vol. 2. Pp. 427-433.

ter Braak C.J.F., and P.F. Verdonschot. 1995. Canonical correspondence analysis and related multivariate methods in aquatic ecology. Aquatic Sciences 57(3): 255-289.

Wood S.N. 2011. Fast stable restricted maximum likelihood and marginal likelihood estimation of semiparametric generalized linear models. Journal of the Royal Statistical Society (B) 73(1):3-36.

Uren V., and A.S. Dadzie. 2015. Public science communication on Twitter: a visual analytic approach. Aslib Journal of Information Management 67(3): 337-355. 
399 Volcovici V., and P.J. Huffstutter. 2017. Trump administration seeks to muzzle U. S. agency

400 employees [online]. Reuters. Available from http://www.reuters.com/article/us-usa-

401 trump-epa-idUSKBN15822X.

402 Walker A. 2017. Alt-government Twitter accounts target Trump [online]. Cable News Network.

403 Available from http://www.cnn.com/videos/politics/2017/01/27/twitter-alt-government-

404 accounts-walker-pkg.cnn.

405 Weisberger M. 2017. "Rogue" science agencies defy trump administration on Twitter:

406 Anonymous accounts are purportedly linked to NASA, National Parks and other agencies

407 [online]. Scientific American. Available from

408 https://www.scientificamerican.com/article/ldquo-rogue-rdquo-science-agencies-defy-

409 trump-administration-on-twitter/.

410 Wilkinson C., and E. Weitkamp. 2013. A case study in serendipity: environmental researchers

411 use of traditional and social media for dissemination. PLoS One 8(12): e84339.

412 Wilson M.J., T.L. Ramey, M. R. Donaldson, R.R. Germain RR, and E. K. Perkin. 2016.

413 Communicating science: Sending the right message to the right audience. FACETS 1:

414 127-137.

415 Xenopoulos M.A., and P.C. Frost. 2015. The dawn of a new era for the Experimental Lakes

$416 \quad$ Area. Limnology and Oceanography Bulletin 24(3): 85-87. 


\section{List of Figures, Tables, and Appendices}

418 Figure 1. Canonical Correspondence Analysis plots comparing science related word and hashtag 419 use by accounts with percent variance explained by axes in parentheses. Differences in word 420 choice are not significantly explained by account type (alt, pre-official, post-official) (A), but are 421 significantly explained by the agency officially or unofficially represented by accounts (B). 422 Ellipses represent $95 \%$ confidence intervals around the centroid for each group. No ellipse is 423 drawn for Badlands National Park as the official Twitter account did not tweet between 24 Jan 4242016 and 1 Apr 2016.

425

426

427

Figure 2. Total retweets (summed across accounts; A) and retweets per tweet (as means across accounts; B) comparing official and alt accounts between 1 Dec 2016 and 1 Apr 2017 (alt

428 accounts began on 24 Jan 2017). Grey lines are daily values and coloured lines are a three-day moving average for clarity. The decrease is significant for alt accounts, though stronger for absolute retweets as the total number of tweets from alt accounts has also decreased over time. The y-axis is truncated at the moving average maximum for clarity.

Table 1. GAMM results from model averaging. Significance levels are $<0.001 * * *,<0.01 * *$, $<0.05^{*},<0.10$. Total number of models incorporated is given by $s, \mathrm{R}^{2}$ is adjusted $\mathrm{R}^{2}$ and both averaged models had a weight of 1 . The intercept estimate is the effect of mean values for emotions, hashtags, and mentions without links or images (binary factors).

439

440

441

442

443

444 445

446 447

Table 2. The twenty terms most likely to be retweeted by category. Terms were ranked if they occurred in four or more tweets (median number of tweets for terms); RT/T refers to retweets per tweet and $\mathrm{n}$ refers to the number of tweets. For comparison against ambient attention levels, the mean number of retweets per tweet across the corpus was 195 and the median was 27.5

Table S1: All accounts associated with U.S. federal agencies or organizations, officially or unofficially, with total number of tweets (including retweets), followings, followers, and likes.

Table S2: All science-related terms and hashtags found in tweets. Agency-related terms were removed prior to $\mathrm{CCA}$. 
Figure 1

Canonical Correspondence Analyses comparing science related term and hashtag use by accounts.

Percent variance explained by axes is given in parentheses by (a) account type (alt, preofficial, post-official) and (b) agency. Ellipses represent 95\% confidence intervals around the centroid for each group.
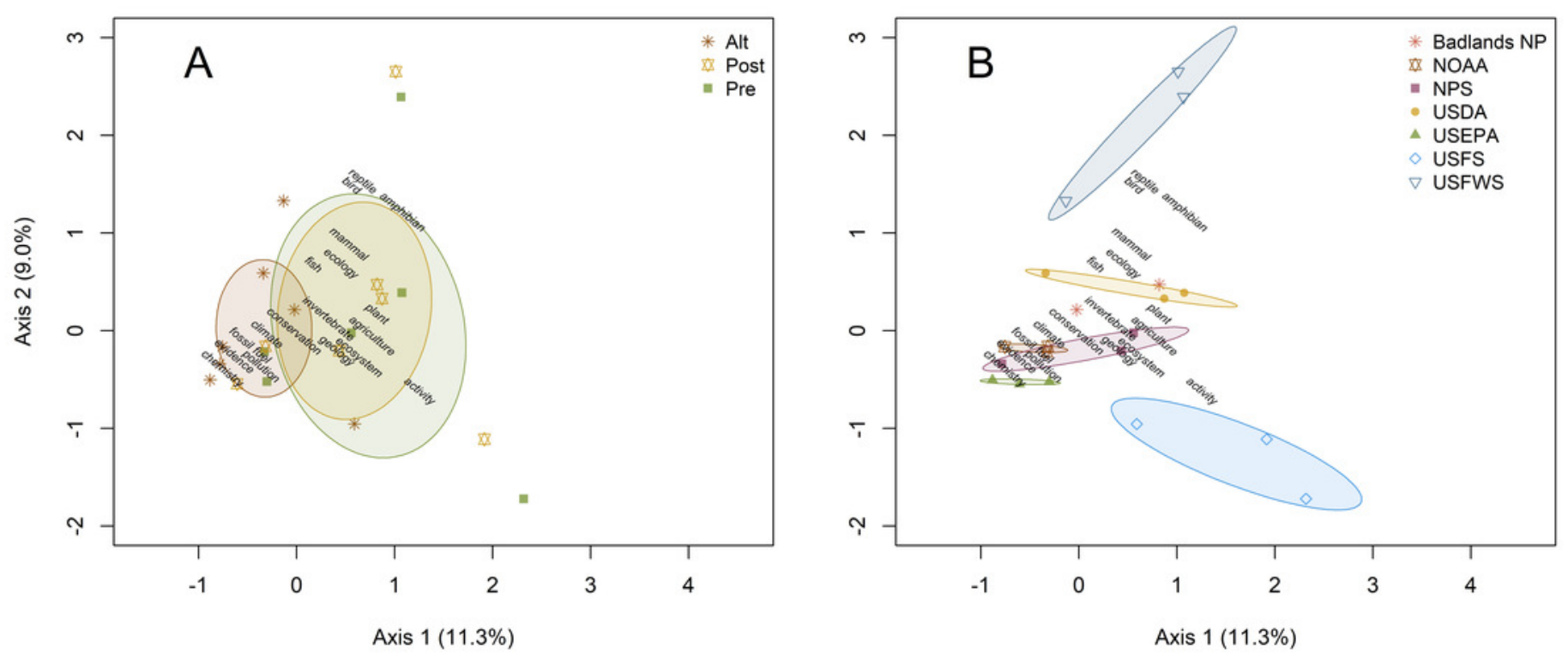
Figure 2

Total retweets summed across all accounts through time.

(a) retweets per tweet (as means across accounts); (b) comparing official and alt accounts between 1 Dec 2016 and 1 Apr 2017 (alt accounts began on 24 Jan 2017). Grey lines are daily values and colored lines are a three-day moving average for clarity. The $y$-axis is truncated at the moving average maximum for clarity. 

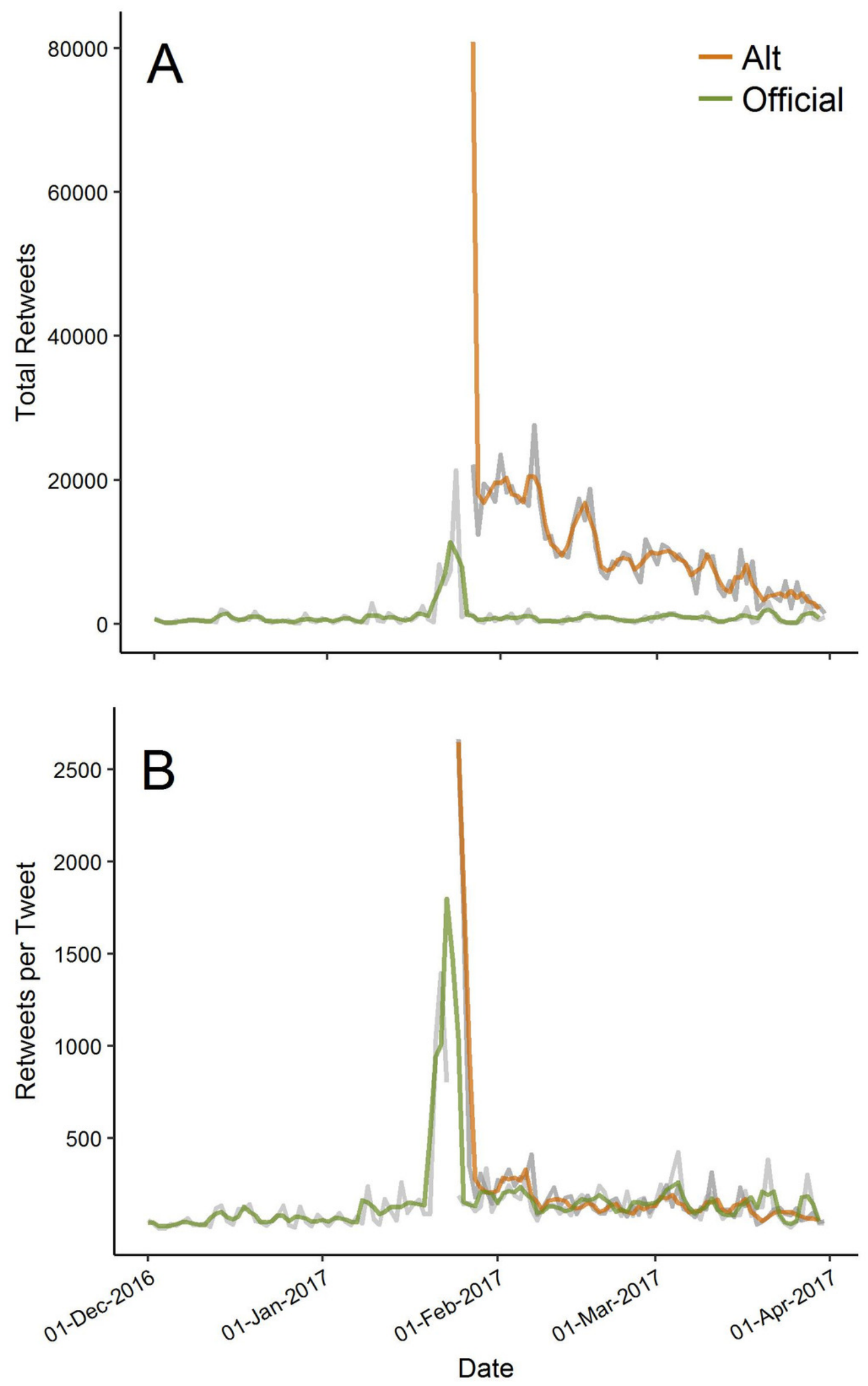

Peer) reviewing PDF | (2021:05:61059:1:1:NEW 6 Sep 2021) 


\section{Table $\mathbf{1}$ (on next page)}

GAMM results from model averaging for mood, tweet features, and accounts.

Significance levels are $<0.001^{* * *},<0.01^{* *},<0.05^{*},<0.10$; total number of models incorporated is given by $s ; R^{2}$ is adjusted $R^{2}$; both averaged models had a weight of 1 ; and the intercept estimate is the effect of mean values for moods, hashtags, and mentions without links or images (binary factors). 


$$
\begin{array}{ll}
\text { Retweets } & \mathrm{R}^{2}=0.59 \\
& s=8
\end{array}
$$

(Intercept)

Mood (Fixed Effects)

$\begin{array}{lll}\text { afraid } & 0.33 & (-0.27 \text { to } 0.93) \\ \text { amused } & -0.22 & (-0.71 \text { to } 0.27) \\ \text { angry } & \mathbf{4 . 4 5} & (3.77 \text { to } 5.13)^{* * *} \\ \text { annoyed } & 0.45 & (-0.36 \text { to } 1.27) \\ \text { happy } & 1.19 & (0.18 \text { to } 2.20) \\ \text { inspired } & \mathbf{- 2 . 9 8} & (-3.69 \text { to }-2.27)^{* * *} \\ \text { sad } & -0.09 & (-0.45 \text { to } 0.27)\end{array}$

Tweet Features (Fixed Effects)

$\begin{array}{lll}\text { Hashtags } & \mathbf{0 . 1 7} & \text { (0.14 to } 0.20)^{* * *} \\ \text { Mentions } & \mathbf{0 . 1 1} & \text { (0.06 to } 0.15)^{*} \\ \text { Links } & \mathbf{0 . 8 2} & \text { (0.77 to } 0.86)^{* * *} \\ \text { Images } & \mathbf{0 . 7 5} & \text { (0.68 to } 0.82)^{* * *}\end{array}$

Account Type (Random Effects)

$\begin{array}{lcl}\text { Alt Account } & 0.69 & (0.04 \text { to } 1.35) \\ \text { Official Post } & 0.21 & (-0.34 \text { to } 0.77) \\ \text { Official Pre } & -0.90 & (-1.46 \text { to }-0.35)\end{array}$

\section{Accounts (Random Effects)}

$\begin{array}{lcl}\text { BadlandsNPS } & 0.72 & (0.25 \text { to } 1.18) \\ \text { BadHombreNPS } & \mathbf{1 . 3 6} & (0.82 \text { to } 1.90)^{*} \\ \text { EPA } & \mathbf{- 1 . 2 0} & (-1.66 \text { to }-0.74)^{* *} \\ \text { altUSEPA } & \mathbf{3 . 4 1} & (2.94 \text { to } 3.89)^{* * *} \\ \text { forestservice } & \mathbf{- 1 . 8 5} & (-2.31 \text { to }-1.38)^{* * *} \\ \text { AltForestServ } & \mathbf{- 1 . 1 5} & (-1.65 \text { to }-0.65)^{*} \\ \text { NatlParkService } & \mathbf{1 . 5 7} & (1.09 \text { to } 2.05)^{* *} \\ \text { AltNatParkSer } & -0.95 & (-1.44 \text { to }-0.45)^{*} \\ \text { NotAltWorld } & -0.24 & (-0.76 \text { to } 0.28) \\ \text { NOAA } & 0.38 & (-0.10 \text { to } 0.86) \\ \text { altNOAA } & -0.60 & (-1.10 \text { to }-0.09) \\ \text { USDA } & 0.02 & (-0.45 \text { to } 0.48) \\ \text { altusda } & -0.29 & (-0.80 \text { to } 0.23) \\ \text { USFWS } & \mathbf{- 1 . 3 2} & (-1.79 \text { to }-0.86)^{* *} \\ \text { AltUSFWS } & 0.14 & (-0.35 \text { to } 0.64)\end{array}$

$$
\begin{array}{cl}
\text { Likes } & \mathrm{R}^{2}=0.66 \\
& s=5 \\
\text { Estimate } & \text { Confidence Interval } \\
\mathbf{4 . 7 5} & (3.98 \text { to } 5.52) * * *
\end{array}
$$

$\begin{array}{ll}-0.07 & (-0.33 \text { to } 0.20) \\ 0.04 & (-0.18 \text { to } 0.26) \\ \mathbf{4 . 1 9} & (3.61 \text { to } 4.76)^{* * *} \\ \mathbf{2 . 1 4} & (1.41 \text { to } 2.88)^{* *} \\ 0.15 & (-0.28 \text { to } 0.58) \\ \mathbf{- 1 . 3 5} & (-1.95 \text { to }-0.75)^{*} \\ -0.12 & (-0.49 \text { to } 0.25)\end{array}$

0.15 (0.12 to 0.17$) * * *$

0.12 (0.07 to 0.16$)^{* *}$

$0.31 \quad(0.27 \text { to } 0.35)^{* * *}$

$\mathbf{0 . 4 4}$ (0.38 to 0.51$)^{* * *}$

$\begin{array}{ll}0.77 & (0 \text { to } 1.53) \\ 0.42 & (-0.25 \text { to } 1.09) \\ -1.18 & (-1.86 \text { to }-0.51)\end{array}$

$0.60 \quad$ (0.13 to 1.07$)$

1.47 (0.94 to 2.00$) * *$

-1.36 (-1.83 to -0.90$) * *$

3.39 (2.92 to 3.87$) * * *$

$\mathbf{- 1 . 6 8}(-2.14 \text { to }-1.22)^{* * *}$

$-0.93 \quad(-1.43$ to -0.43$)$.

1.08 (0.60 to 1.56$)^{*}$

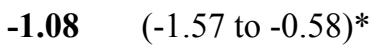

$0.07 \quad(-0.44$ to 0.59$)$

$0.48 \quad$ (0 to 0.95$)$

$-0.70 \quad(-1.21$ to -0.20$)$

$-0.04 \quad(-0.50$ to 0.42$)$

$-0.33 \quad(-0.84$ to 0.18$)$

$\mathbf{- 1 . 2 8}(-1.74 \text { to }-0.81)^{* *}$

$0.32 \quad(-0.18$ to 0.81$)$ 


\section{Table 2 (on next page)}

The twenty terms most likely to be retweeted by category.

Terms were ranked if they occurred in four or more tweets (median number of tweets for terms); RT/T refers to retweets per tweet and $\mathrm{n}$ refers to the number of tweets. 


\begin{tabular}{|c|c|c|c|c|c|c|c|c|c|c|c|c|}
\hline \multirow[b]{2}{*}{ Rank } & \multicolumn{3}{|c|}{ Ecosystem } & \multicolumn{3}{|c|}{ Evidence } & \multicolumn{3}{|c|}{ Organism } & \multicolumn{3}{|c|}{ Climate Change } \\
\hline & Term & $\begin{array}{l}\text { RT/ } \\
\text { T }\end{array}$ & $\mathrm{n}$ & Term & $\begin{array}{l}\text { RT/ } \\
\text { T }\end{array}$ & $\mathrm{n}$ & Term & $\begin{array}{l}\mathrm{RT} / \\
\mathrm{T}\end{array}$ & $\mathrm{n}$ & Term & $\begin{array}{l}\mathrm{RT} / \\
\mathrm{T}\end{array}$ & $\mathrm{n}$ \\
\hline 1 & prairie & 1248 & 13 & $\begin{array}{l}\text { peer- } \\
\text { reviewed }\end{array}$ & 2804 & 7 & violets & 904 & 4 & keystone & 5326 & 4 \\
\hline 2 & forest & 940 & $\begin{array}{l}14 \\
8\end{array}$ & conference & 2579 & 4 & mammal & 665 & 10 & spill & 1671 & 6 \\
\hline 3 & plains & 523 & 6 & knowledge & 2486 & 10 & rose & 621 & 9 & diesel & 1304 & 6 \\
\hline 4 & streams & 440 & 12 & verify & 2156 & 6 & bison & 613 & 7 & atmosphere & 1007 & 18 \\
\hline 5 & ecosystem & 343 & 8 & \#antiscience & 2044 & 6 & wolf & 460 & 12 & \#standingrock & 960 & 5 \\
\hline 6 & reef & 320 & 8 & denial & 1968 & 20 & otter & 452 & 4 & oil & 884 & 30 \\
\hline 7 & sea & 234 & 25 & censorship & 1902 & 10 & coral & 441 & 5 & \#allpipesleak & 880 & 4 \\
\hline 8 & coast & 232 & 6 & evidence & 1701 & 18 & fish & 348 & 11 & climate & 813 & 226 \\
\hline 9 & arctic & 218 & 9 & study & 1145 & 15 & animal & 301 & 87 & \#nodapl & 795 & 21 \\
\hline 10 & ocean & 177 & 29 & scientific & 1133 & 50 & chicken & 286 & 8 & pipeline & 725 & 26 \\
\hline 11 & $\begin{array}{l}\text { \#worldwetlandsd } \\
\text { ay }\end{array}$ & 163 & 6 & facts & 897 & 95 & shark & 266 & 5 & \#nokxl & 697 & 4 \\
\hline 12 & lake & 127 & 12 & investigate & 715 & 6 & eagle & 233 & 16 & dioxide & 674 & 15 \\
\hline 13 & beach & 125 & 7 & accurate & 547 & 7 & owl & 233 & 15 & carbon & 578 & 23 \\
\hline 14 & woods & 109 & 9 & data & 338 & 62 & chick & 216 & 7 & \#climatechange & 478 & 66 \\
\hline 15 & wetland & 105 & 13 & think & 271 & 113 & seal & 194 & 10 & \#dapl & 438 & 8 \\
\hline 16 & river & 100 & 33 & analysis & 253 & 5 & eaglet & 186 & 4 & fracking & 303 & 11 \\
\hline 17 & island & 95 & 8 & published & 223 & 4 & tree & 172 & 48 & drilling & 296 & 9 \\
\hline 18 & desert & 81 & 4 & explain & 180 & 21 & $\begin{array}{l}\text { \#dceagleca } \\
\mathrm{m}\end{array}$ & 171 & 7 & temperature & 249 & 7 \\
\hline 19 & cuyahoga & 71 & 4 & proof & 156 & 6 & fox & 168 & 21 & $\mathrm{CO}_{2}$ & 209 & 30 \\
\hline 20 & \#forestfriday & 62 & 4 & $\begin{array}{l}\text { \#climatefact } \\
\mathrm{s}\end{array}$ & 149 & 41 & albatross & 166 & 5 & $\begin{array}{l}\text { \#climatechangeisre } \\
\text { al }\end{array}$ & 196 & 16 \\
\hline
\end{tabular}

